\title{
A practical trial to use Thyroid Imaging Reporting and Data System (TI-RADS) in differentiation between benign and malignant thyroid nodules
}

\author{
Enas A. Azab * (D), Ahmad S. Abdelrahman and Marwa Elsayed Abdelrahman Ibrahim
}

\begin{abstract}
Background: Thyroid nodules have been reported up to 68\% in adults using ultrasound examination. The American College of Radiology Thyroid Imaging Reporting and Data System (ACR TI-RADS) is founded on the evaluation of ultrasound (US) features in five categories: composition, echogenicity, shape, margin, and echogenic foci; the nodule's total points determine its risk level, which ranges from TI-RADS1 (TR1) (benign) to TI-RADS5 (TR5) (highly suspicious). In conjunction with the nodule's maximum diameter, the TR level determines whether to recommend a fine-needle aspiration (FNA) biopsy, a follow-up US examination, or no further action; our aim in this study was to apply ACR TI-RADS as a method for discrimination between benign and malignant nodules.

Methods: We applied ACR TI-RADS template for 40 thyroid nodules, the total points given to thyroid nodules ranged from 0 to 14 points, then we compared our results with scintigraphy, fine-needle aspiration cytology (FNAC), and histopathological reports after surgery.

Results: Of 40 thyroid nodules, 31 nodules (77.5\%) were benign and 9 nodules (22.5\%) were malignant; there was a statistically high significant trend of increasing risk of malignancy as the final TI-RADS level increased from TR1 to TR5 ( $P$ value $<0.001)$.

Conclusion: The ACR TI-RADS scoring system is a simple easy method to be applied in daily ultrasound practice; it has an excellent diagnostic accuracy for the diagnosis of malignant thyroid nodules in the present study, as the aggregate risk of malignancy increased as the TI-RADS level increases from TR1 to TR5.
\end{abstract}

Keywords: The American College of Radiology (ACR), Thyroid Imaging Reporting and Data System (TI-RADS, TR), Ultrasound (US)

\section{Background}

The American College of Radiology (ACR) Thyroid Imaging Reporting and Data System (TI-RADS) is a reporting system for the evaluation of thyroid nodule by ultrasound (US) to determine management [1]. ACR TIRADS is founded on the evaluation of five categories seen by the US, this includes composition, echogenicity, shape, margin, and echogenic foci; each feature is assigned $0-3$ points. Features in the first four categories each have a single score, whereas more than one feature may be present in the echogenic foci category. The nodule's total points determine its risk level from TR1 (benign) to TR5 (highly suspicious) (Table 1). In conjunction with the nodule maximum diameter, the TR level determines whether to recommend fine-needle aspiration (FNA), biopsy, a follow-up US examination, or no further action [2] (Table 2). Our aim of this study was to apply ACR TI-RADS stratification system as a method for discrimination between benign and malignant thyroid nodules.

* Correspondence: enasazab@yahoo.com

Radiology Department, Faculty of Medicine, Ain Shams University, Cairo, Egypt 
Table 1 TI-RADS level

\begin{tabular}{lll}
\hline TI-RADS level & The sum of the points & Classification \\
\hline TR1 & 0 & $\begin{array}{l}\text { Benign } \\
\text { Not } \\
\text { TR2 }\end{array}$ \\
TR3 & 3 & $\begin{array}{l}\text { Mildly } \\
\text { suspicious }\end{array}$ \\
TR4 & 4 to 6 & $\begin{array}{l}\text { Moderately } \\
\text { suspicious } \\
\text { TR5 }\end{array}$ \\
& 7 or more than 7 & $\begin{array}{l}\text { Highly } \\
\text { suspicious }\end{array}$ \\
\hline
\end{tabular}

\section{Image analysis \\ Composition}

Composition refers to the presence of soft tissue or fluid, and the proportion of each. Spongiform nodules refer to the presence of tiny cysts resembling fluid-filled spaces in a wet sponge, and at least $50 \%$ of the nodule volume should be occupied by these cysts [3, 4]. In ACR TIRADS, spongiform nodules are benign, with no further follow-up. Solid nodules are composed entirely or near entirely of soft tissue. Partially solid and partially cystic nodules are determined according to the percentage of the solid component which is highly subjective [2].

\section{Echogenicity}

Echogenicity describes the level of echogenicity of the solid, non-calcified component of a nodule, compared to the thyroid parenchyma used as a reference, except for very hypoechoic nodules, in which the strap muscles are used as the basis for comparison [5]. If the nodule is of mixed echogenicity, it can be described as "predominantly" hyperechoic, isoechoic, or hypoechoic [6].

\section{Margin}

Smooth margin is characterized by an even curvilinear edge. Lobulation and angulation both warrant two points. Extrathyroidal extension takes three points which is pathognomonic for malignancy. If the nodule's border

Table 2 Triage of nodules for FNA or observation [1]

\begin{tabular}{ll}
\hline TI-RADS level & Recommendations \\
\hline TR1 & No FNA required \\
TR2 & No FNA required \\
TR3 & Nodule size $\geq 1.5 \mathrm{~cm}$ : follow-up \\
& after 1,3, and 5 years \\
& Nodule size $\geq 2.5 \mathrm{~cm}$ : FNA is \\
& recommended \\
& Nodule size $\geq 1.0 \mathrm{~cm}$ : follow-up after \\
& $1,2,3$, and 5 years \\
TR4 & Nodule size $\geq 1.5 \mathrm{~cm}:$ FNA \\
& is recommended \\
& Nodule size $\geq 0.5 \mathrm{~cm}:$ annual follow-up \\
& for up to 5 years \\
TR5 & Nodule size $\geq 1.0 \mathrm{~cm}:$ FNA is recommended \\
&
\end{tabular}

is not clear, it is categorized as ill-defined and receives zero points.

\section{Presence of echogenic foci}

The presence of echogenic foci is seen in both benign and malignant lesions. They vary in size and shape and are divided into the following categories. Absence of echogenic foci or the presence of large comet tail artifacts is assigned zero points. Large comet Vshaped artifact of more than one $\mathrm{mm}$ is associated with colloids and is a reliable sign of benignity when found in the cystic components of nodules. On the other hand, small comet-tail artifacts should be treated as punctate echogenic foci (PEF) and therefore receive three points. If both types coexist in a nodule, the more suspicious one determines how many points to assign [2]. Macrocalcification is assigned one point. Peripheral (rim) calcifications are assigned two points. The presence of punctate echogenic foci is assigned three points. Because different echogenic foci can coexist in the same nodule, the points of all echogenic foci can be added together [1].

\section{Shape}

Shape means wider than tall or taller than wide on an axial image. The goal is to ascertain whether the nodule has grown more front to back than side to side, which suggests that it has violated tissue planes and is therefore suspicious [7].

\section{Methods}

A cross-sectional study was conducted at Ain Shams University hospitals from October 2017 to March 2018. Our study was approved by the ethical committee from the Radiology Department, Faculty of Medicine, Ain Shams University. The number of patients was 40. The age group ranges from 25 to 69 years old. The inclusion criteria were patients with one or more thyroid nodules that meet the TI-RADS template criteria, with no sex predilection. Exclusion criteria are as follows: patients with thyroid nodule less than one $\mathrm{cm}$, patients with bleeding tendency, and pregnant patients. Full explanation of the procedure was given and written consent from all patients was obtained. Ultrasound of the thyroid gland was performed in three ultrasound machines (LOGIQ $^{\mathrm{Tm}}$ P7 and P9, GE Healthcare and Accuvix XG, Samsung) using a high-frequency probe (7-10 MHz). Axial and sagittal ultrasound scanning of the thyroid nodules was performed with the patient supine and head slightly extended. Imaging interpretation of thyroid nodules was done, guided by the American College of Radiology (ACR) Thyroid Imaging, Reporting and Data System (TI-RADS) Criteria including evaluation of five 


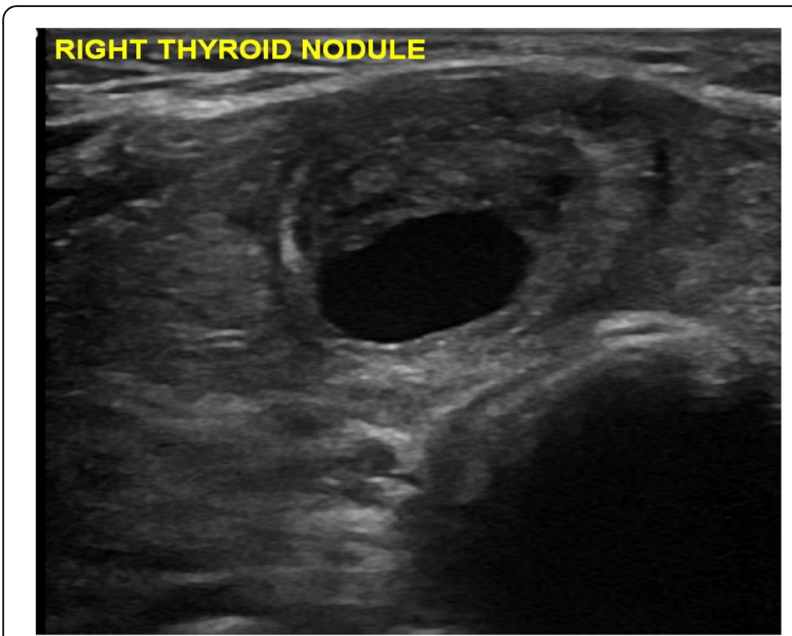

Fig. 1 Female patient with hypoechoic mixed composition right thyroid nodule TR3. FNA revealed benign nodular hyperplasia with cystic degeneration

categories: composition, echogenicity, shape, margin, and echogenic foci.

To confirm the nature of the scanned thyroid nodules (either benign or malignant), one or more of the following were done:

1. Fine-needle aspiration cytology (FNAC) report: FNAC was performed using a 26-gauge needle attached to a $10-\mathrm{ml}$ syringe; two or three aspirations were performed on each nodule.

2. Surgical histopathology report

3. Thyroid scintigraphy results: where different neck image views were obtained using a gamma camera, 20 min after intravenous injection of 3$5 \mathrm{mCi}$ of technetium $99 \mathrm{~m}$ pertechnetate, while the patient was supine and hyperextended.

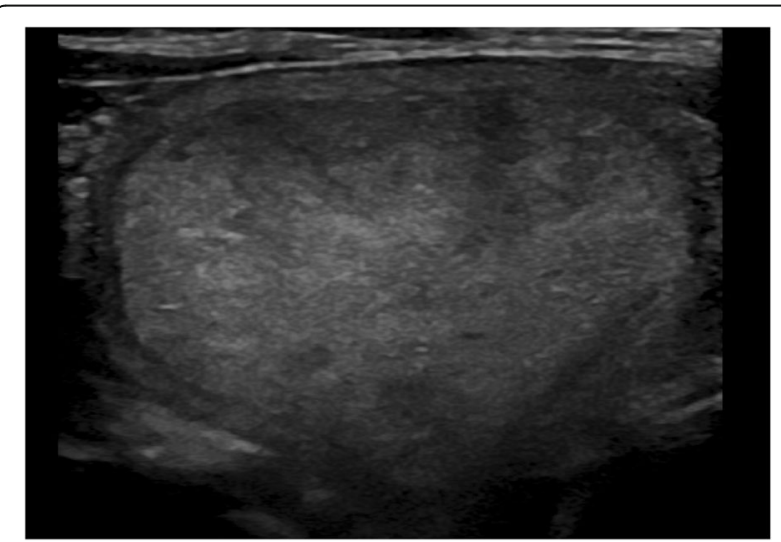

Fig. 2 TR3 US revealed a hyperechoic wider than taller solid nodule with a well-defined margin, no echogenic foci was seen inside, and the histopathology revealed adenoma

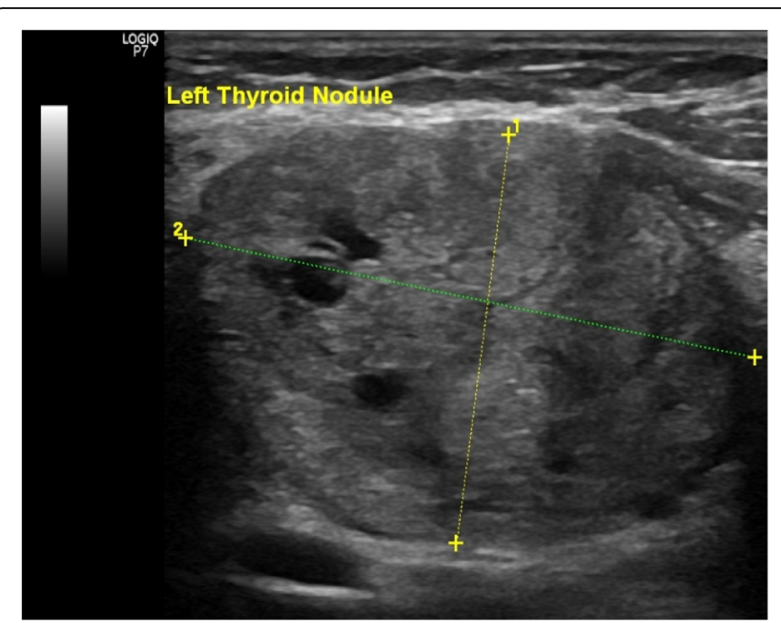

Fig. 3 Female patient with a solid hypoechoic nodule wider than taller, no calcification, and smooth margin TR4; FNAC revealed Hashimoto's thyroiditis

Statistical analysis was done using Statistical Package for the Social Sciences (IBM SPSS) version 23. The quantitative data were presented as mean, standard deviations, and ranges when their distribution was found parametric while with non-parametric distribution they were presented as median with inter-quartile range (IQR). The comparison between two independent groups with quantitative data and parametric distribution was done by using an independent $t$-test while with non-parametric distribution they were compared by using a Mann-Whitney test. The $P$ value $<0.01$ was considered highly significant.

\section{Results}

A total of 40 patients was included in the study, seven males (17.5\%) and 33 females (82.5\%). The mean patient

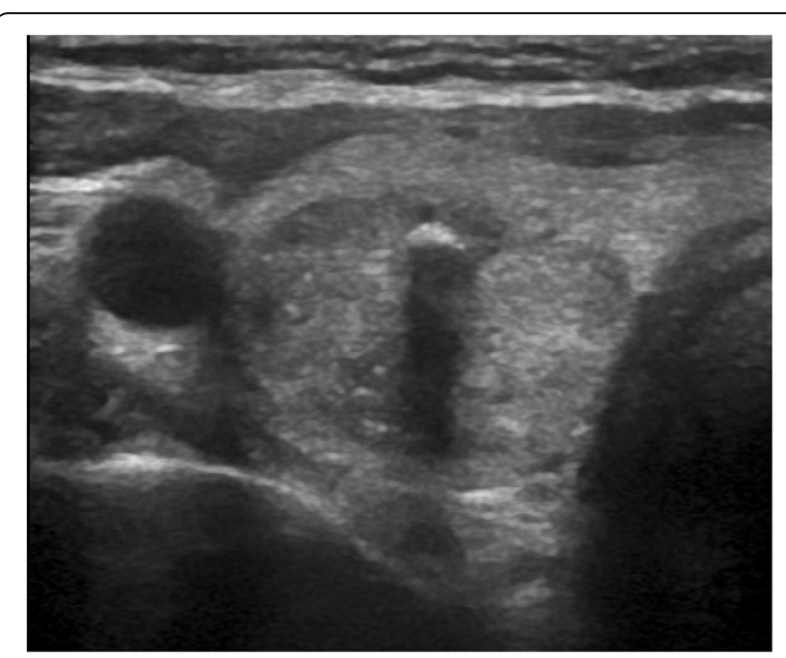

Fig. 4 Solitary thyroid nodule was solid hypoechoic with macrocalcification and punctate calcification TR5; papillary carcinoma was evident after surgical removal 

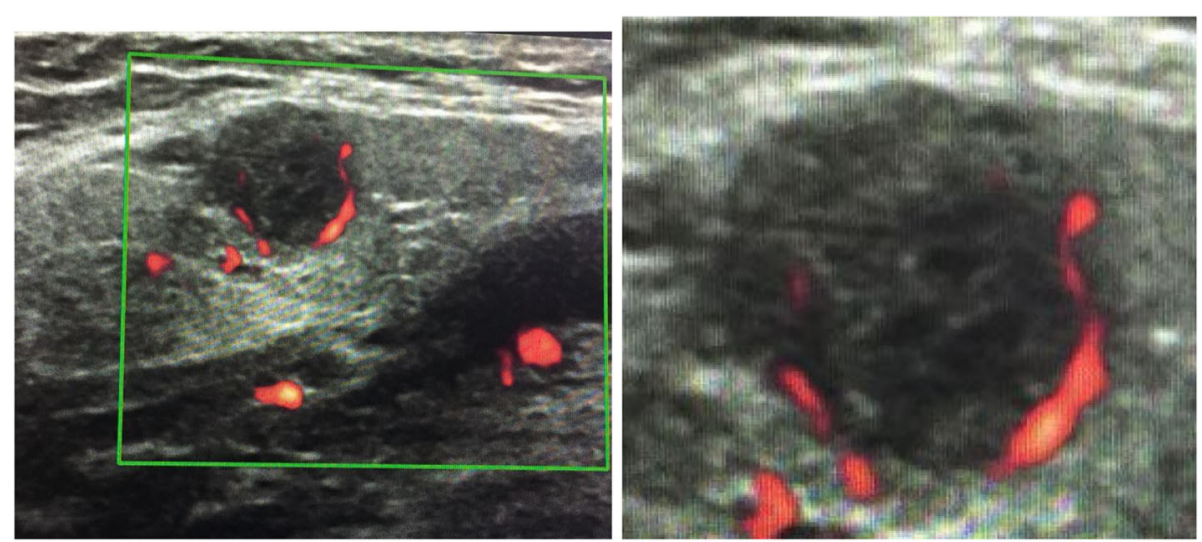

Fig. 5 Transverse and longitudinal scan revealed a very hypoechoic solid nodule with an irregular margin and fine echogenic foci seen inside TR5; the histopathology revealed follicular variant of papillary carcinoma

age was $44.48 \pm 11.04$ years old (range $25-69$ years). 21 patients $(52.5 \%)$ had a solitary nodule while 19 patients (47. 5\%) had more than one nodule (the thyroid nodule with the highest TI-RADS score was included in the study). Out of the 40 nodules included in the study, 31 nodules $(77.5 \%)$ were benign and nine nodules $(22.5 \%)$ were malignant nodules. Regarding benign nodules, scintigraphy done for five patients revealed two autonomous functioning nodules and three toxic multinodular goiters; cytology in 22 patients revealed eight colloid cysts/goiters, five colloid cysts with hemorrhage, two hemorrhagic cysts, and seven benign nodular hyperplasia (Fig. 1); and cytology followed by surgical histopathology in three patients revealed two benign multinodular goiters and one follicular adenoma (Fig. 2). Surgical histopathology done for one patient proved nodular Hashimoto's thyroiditis (Fig. 3).

Regarding malignant nodules, cytology in 4 patients revealed 3 papillary carcinomas and 1 with atypical malignant cells (Fig. 4). Cytology followed by surgical histopathology was done for 3 patients and revealed 2 papillary carcinoma and 1 follicular variant of papillary carcinoma. Surgical histopathology was done for 2 patients and revealed 1 micropapillary carcinoma and 1 follicular carcinoma (Fig. 5) (Table 3).

The total points given to the thyroid nodules included in the study ranged from zero to 14 points. Our study showed that there was a statistically highly significant increase in the incidence of solid thyroid nodules, very hypoechoic thyroid nodules, taller than wider nodules, nodules having lobulated or irregular margins, nodules having extrathyroidal extension, and thyroid nodules with punctate echogenic foci in the malignant group than in the benign group with $P$ values $0.001,0.001$, $0.001,{ }^{<} 0.001,0.007$, and ${ }^{<} 0.001$ respectively (Table 4 ).

On the other hand, there was statistically high significant increase in the incidence of wider than taller nodules in the benign group than in the malignant group with $P$ value $=0.001$, and there was statistically significant increase in the incidence of cystic, mixed cystic and solid thyroid nodules, nodules having smooth margin, nodules having ill-defined margin, nodules with no calcifications, and thyroid nodules with large comet tail artifact in the benign group than in the malignant group with $P$ values $0.018,0.018,0.005,0.036,0.02$, and 0.049 respectively (Table 4 ).

Our study showed that there was no statistically significant difference found between the benign and the malignant groups regarding the spongiform thyroid nodules, the nodules with isoechoic or hypoechoic texture, nodules with macrocalcifications, and nodules with peripheral calcifications.

The median of total points for the benign group was three with IQR from zero to eight while the median of

Table 3 Classification of the 40 thyroid nodules

\begin{tabular}{lc}
\hline 40 Thyroid nodules & \\
\hline Benign (no. $=31)$ & Malignant (no. $=9$ ) \\
5 confirmed by & 4 confirmed by cytology: \\
thyroid scintigraphy: & 3 papillary carcinoma \\
2 autonomous & 1 atypical malignant cell \\
functioning nodules & \\
3 toxic multinodular goiters & \\
22 confirmed by cytology: & 3 confirmed by \\
8 colloid cysts/goiters & cytology followed by surgical \\
5 colloid cysts with hemorrhage & histopathology: \\
2 hemorrhagic cysts & 2 papillary carcinoma \\
7 benign nodular hyperplasia & 1 follicular variant of \\
& papillary carcinoma \\
3 confirmed by cytology followed & 2 confirmed by \\
by surgical histopathology: & surgical histopathology: \\
2 benign multinodular goiter & 1 micropapillary \\
1 follicular adenoma & carcinoma \\
1 confirmed by surgical & 1 follicular carcinoma \\
histopathology: & \\
Nodular Hashimoto's thyroiditis & \\
\hline
\end{tabular}


Table 4 Comparison between the benign group and malignant group regarding composition, echogenicity, shape, margins of thyroid nodules, and presence of echogenic foci in the thyroid nodules

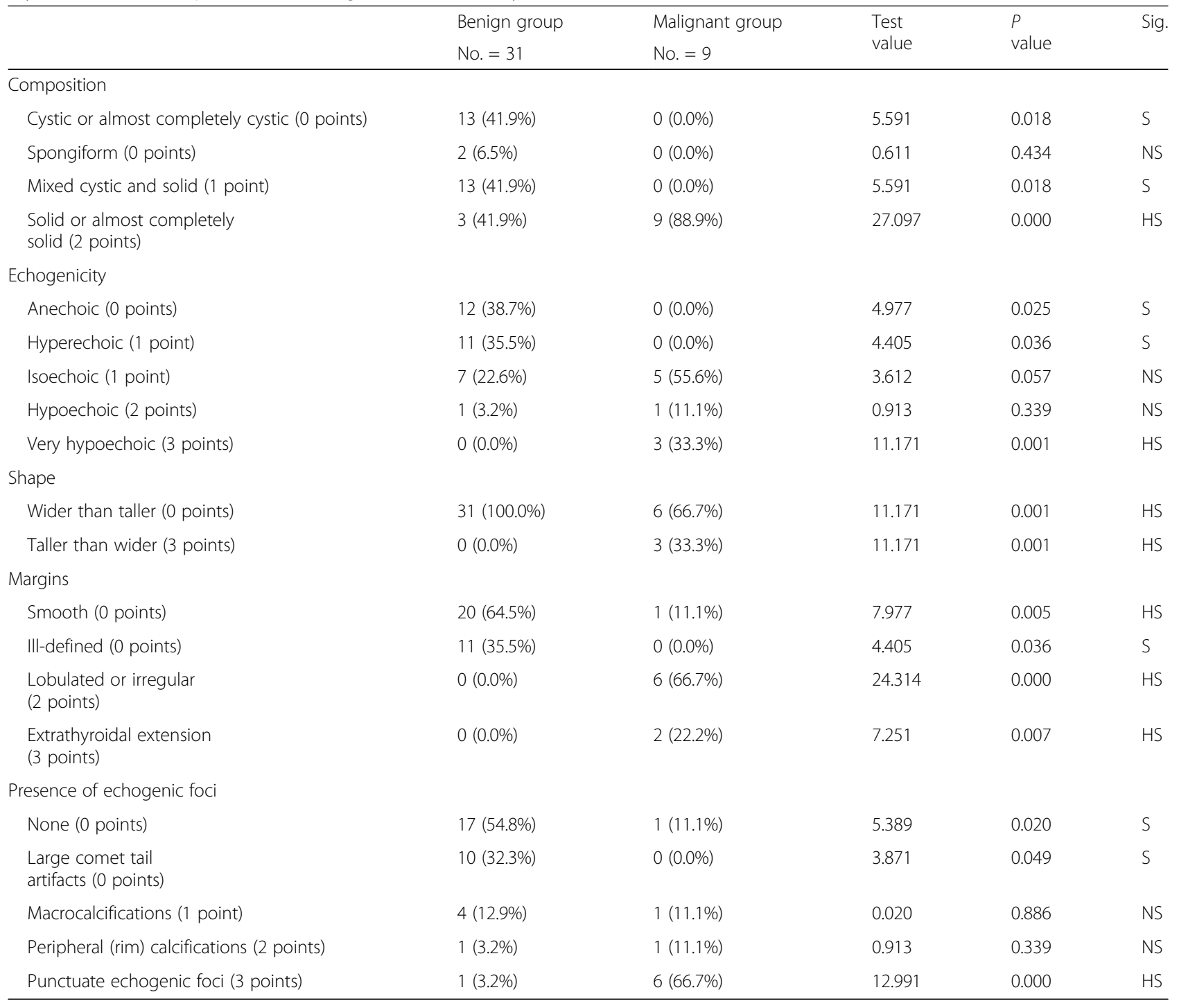

total points for the malignant group was eight IQR from five to 13 (Table 5). The median of TI-RADS score for the benign group was TR 3 with IQR from one to five while the median of TI-RADS score for the malignant group was TR 5 with IQR from four to five. The risk of malignancy for TR4 and TR5 nodules was 11.1\% and $66.6 \%$ respectively (Fig. 6). In our study, the best cut off point to detect malignant cases was five which corresponds to TR4 with a sensitivity of $88.89 \%$, specificity $96.77 \%$, positive predictive value (PPV) of $88.9 \%$, and

Table 5 Comparison between benign group and malignant group regarding total points and TI-RADS level

\begin{tabular}{|c|c|c|c|c|c|c|}
\hline & & \multirow{2}{*}{$\begin{array}{l}\text { Benign group } \\
\text { No. }=31\end{array}$} & Malignant group & \multirow{2}{*}{$\begin{array}{l}\text { Test } \\
\text { value }\end{array}$} & \multirow{2}{*}{$\begin{array}{l}P \\
\text { value }\end{array}$} & \multirow[t]{2}{*}{ Sig. } \\
\hline & & & No. $=9$ & & & \\
\hline \multirow[t]{2}{*}{ Total points } & $\begin{array}{l}\text { Median } \\
\text { (IQR) }\end{array}$ & $3(0-8)$ & $8(5-13)$ & -7.170 & 0.000 & HS \\
\hline & Range & $0-8$ & $5-13$ & & & \\
\hline \multirow[t]{2}{*}{ TI-RADS level } & $\begin{array}{l}\text { Median } \\
\text { (IQR) }\end{array}$ & $3(1-5)$ & $5(4-5)$ & -5.387 & 0.000 & HS \\
\hline & Range & $1-5$ & $4-5$ & & & \\
\hline
\end{tabular}




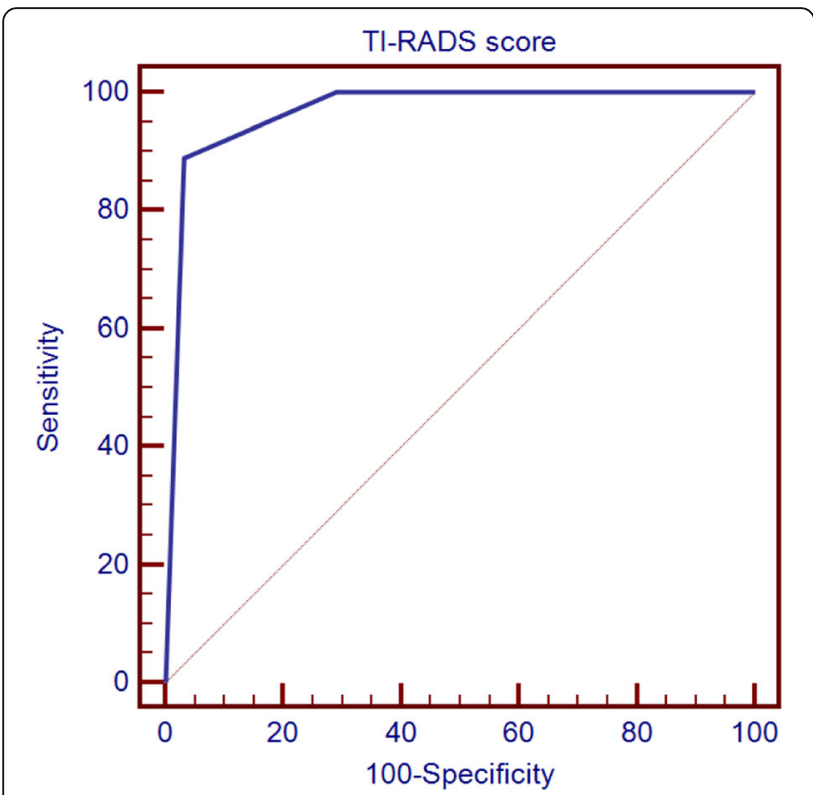

Fig. 6 ROC curve demonstrating the diagnostic performance of the TI-RADS level. It shows that the best cut off point to detect malignant cases regarding TI-RADS score found TR5 with a sensitivity of $88.89 \%$, specificity of $96.77 \%$, and area under curve (AUC) of $96.8 \%$

negative predictive value (NPP) of $69.8 \%$. There was a statistically high significant trend of increasing risk of malignancy as the total points increased $(P$ value < 0.001 ) and as the final TI-RADS level increased from TR1 to TR5 ( $P$ value $<0.001)$ (Table 6$)$.

\section{Discussion}

Ultrasound is the most commonly used imaging technique in the evaluation of thyroid nodules so it is essential to establish a lexicon that provides a structured method for evaluation of thyroid nodules whether the benign or the malignant group [6]. ACR TI-RADS is used as a guideline in stratification of thyroid nodules on the basis of their risk of malignancy [8]. The ACR TI-RADS guidelines were developed to facilitate a standard approach to assess nodular features and to decrease the variation seen in reporting of thyroid nodules in current practice among interpreters with varying levels of sonographic expertise and also to develop guidelines for further management. Five items were assigned (composition, echogenicity, shape, margin, and presence of echogenic foci) and the total points are summed to determine the overall TI-RADS level that ranges from one

Table 6 The diagnostic performance of TI-RADS level

\begin{tabular}{llllll}
\hline Cut off TI-RADS level & AUC & Sensitivity & Specificity & PPV & NPV \\
\hline 5 & 0.968 & 88.89 & 96.77 & 88.9 & 96.8 \\
\hline
\end{tabular}

to five according to the risk of malignancy. For each level, a specific size cut off for FNA is recommended [1].

Our study included 40 thyroid nodules, and ultrasonographic appearance of the thyroid nodules was interpreted using a ACR TI-RADS template to determine whether they belong to the benign or the malignant group. Allocation of points for different sonographic findings mirrors the likelihood that those finding are associated with malignancy; these data were confirmed finally by scintigraphy and histopathological reports after FNA or surgical excision.

Malignancy is uncommon with predominantly cystic nodules [8]. Also, Ahn et al. [9] found that thyroid cancers are more likely to be solid or nearly entirely solid. In our study, all nodules with cystic changes (predominantly cystic) were benign, and there was a highly significant increase in the incidence of solid nodules in the malignant group than in the benign group $(P$ value $<0.001)$.

Regarding echogenicity, there were many studies that showed that the risk of malignancy is inversely proportional to nodule echogenicity; the more echogenicity, the less possibility of malignancy. Papillary and medullary thyroid cancer appears hypoechoic due to increased cellular impaction [10]. The exception to this theory is the follicular dominant pathology which is composed of small microfollicles and tends to appear hyperechoic $[5,11,12]$. In our study, there was a highly significant increase in anechoic and hyperechoic nodules in the benign group $(P$ value $=0.025$ and 0.036 respectively), while there was high increased incidence of very hypoechoic nodules in the malignant group $(P$ value $=0.001)$ and there was no significant difference between the benign and the malignant groups regarding isoechoic and hypoechoic thyroid nodules; this could be related to the histological subtypes of neoplasms included in the study population rather than true frequency.

Middleton et al. [8] found that $12.9 \%$ of nodules with smooth margins and $44.7 \%$ of nodules with a lobulated or irregular border were malignant. Irregular and lobulated margins are suspicious for thyroid malignancy [13]. We had comparable results in our study; we found that $11.1 \%$ of the malignant group nodules had smooth margins and $66.7 \%$ of the malignant group nodules had lobulated or irregular margins. It is important to assess extrathyroidal extension when evaluating thyroid malignancy. In our study, extrathyroidal extension was detected in two patients.

Regarding the shape, thyroid cancer is associated with nodules with a ratio of anteroposterior to transverse diameter greater than one in the transverse view. Specificity ranges from 82 to $93 \%$; this means that it is a less suspicious nodule $[9,14]$. In agreement with these 
previous studies, we found that there was a statistically high significant increase in the incidence of taller than wider nodules in the malignant group than in the benign group with $P$ value 0.001

Middleton et al. [8] found that the risk of malignancy associated with peripheral calcifications, and punctate echogenic foci in solid nodules was $20.2 \%$ and $35 \%$ respectively; we had comparable results to those reported as we found that $11.1 \%$ of the malignant nodules had peripheral calcifications and $66.7 \%$ of the malignant nodules had punctate echogenic foci. Our study also agreed with Reading et al. [15] that macrocalcifications were found within both benign and malignant nodules yet more in the benign nodules.

Regarding the diagnostic accuracy of TI-RADS scoring system studied by Tressler et al. [1], they found an increased risk of malignancy in thyroid nodules starting from TR3 (5\%) to TR4 and TR5 (20\%). Another study done by Middleton et al. [8] stated that the risk of malignancy is $4.8 \%, 9.1 \%$, and $35 \%$, for TR 3, TR4, and TR5 respectively.

In our study, we found that the best cut off point to detect malignant cases was 5 (corresponding to TIRADS level 4) with a sensitivity of $88.89 \%$, specificity of $96.77 \%$, PPV of $88.9 \%$, and NPV of $69.8 \%$. A statistically significant trend of an increasing risk of malignancy was noted as the total points increased $(P$ value $<0.001)$ and as the final TI-RADS level increased from TR1 to TR5 $(P$ value $<0.001)$ (Table 6).

\section{Conclusion}

The ACR TI-RADS scoring system is a simple and practical method for assessing thyroid nodules. It is easy to be applied in daily ultrasound practice, and it also has shown an excellent diagnostic accuracy for the diagnosis of malignant thyroid nodules in the present study. TI-RADS gives the surgeon an idea about the following step (followup(biopsy) as the aggregate risk of malignancy increased as the TI-RADS level increases from TR1 to TR5.

\section{Study limitations}

Our study was limited by the small number of patients; also, other features that are not included in the ACR TIRADS template such as the presence of peripheral halo, pattern of nodular vascularity, and the emerging ultrasound elastography techniques which may help in better characterization of other features of thyroid nodules were not evaluated in our study.

\section{Abbreviations}

ACR: The American College of Radiology; FNA: Fine-needle aspiration; FNAC: Fine-needle aspiration cytology; IQR: Inter-quartile range; NPV: Negative predictive value; PEF: Punctuate echogenic foci; PPV: Positive predictive value; SPSS: Statistical Package for the Social Sciences; TIRADS: Thyroid Imaging Reporting and Data System; US: Ultrasound

\section{Acknowledgements}

Not applicable

\section{Authors' contributions}

EA has performed ultrasound examination and carried out interpretation of US images. She has participated in data collection and writing the manuscript. AA has performed ultrasound examination and carried out interpretation of US images. He has participated in data collection and helped to draft the manuscript. MI has participated in the data collection, conceived of the study, and helped to draft the manuscript. All authors read and approved the final manuscript.

\section{Funding}

No funds, sponsorship, or financial support to be disclosed.

\section{Availability of data and materials}

The datasets used and/or analyzed during the current study are available from the corresponding author on reasonable request.

\section{Ethics approval and consent to participate}

This study was approved by the Research Ethics Committee of the Faculty of Medicine at Ain Shams University in Egypt on July 2017. All patients included in this study gave written informed consent to participate in this research. If the patient was less than 16 years old or unconscious at the time of the study, written informed consent for their participation was given by their parent or legal guardian.

\section{Consent for publication}

All patients included in this research gave written informed consent to publish the data contained within this study. If the patient was less than 16 years old, deceased, or unconscious when consent for publication was requested, written informed consent for the publication of this data was given by their parent or legal guardian.

\section{Competing interests}

We agree that all copyright ownership is transferable to the Association of University Radiologists when the manuscript is accepted for publication in EJRNM. We certify that the submitted article will not constitute "Redundant Publication". This study is not previously published nor submitted elsewhere and the methods employed respect the Helsinki Declaration of 1975, as revised in 1983. The authors declare that they have no competing interests.

Received: 29 June 2019 Accepted: 22 July 2019

Published online: 06 September 2019

\section{References}

1. Tessler FN, Middleton WD, Grant EG et al (2017) ACR thyroid imaging reporting and data system (TI-RADS): white paper of the ACR TI-RADS committee. J Am Coll Radiol 14(5):587-595

2. Tessler FN, Middleton WD, Grant EG (2018) Thyroid Imaging Reporting and Data System (TI-RADS): A User's guide. Radiology. 287(1):29-36

3. Moon WJ, Jung SL, Lee JH et al (2008) Benign and malignant thyroid nodules: US differentiation-multicenter retrospective study. Radiology 247(3):762-770

4. Bonavita JA, Mayo J, Babb J et al (2009) Pattern recognition of benign nodules at ultrasound of the thyroid: which nodules can be left alone? AJR Am J Roentgenol 193(1):207-213

5. Kwak JY, Han KH, Yoon JH et al (2011) Thyroid imaging reporting and data system for US features of nodules: a step in establishing better stratification of cancer risk. Radiology 260:892-899

6. Grant EG, Tessler FN, Hoang JK et al (2015) Thyroid ultrasound reporting lexicon: white paper of the ACR Thyroid Imaging, Reporting and Data System (TIRADS) Committee. J Am Coll Radiol. 12(12 PT A):1272-1279

7. Kim EK, Park CS, Chung WY et al (2002) New sonographic criteria for recommending fine-needle aspiration biopsy of nonpalpable solid nodules of the thyroid. AJR Am J Roentgenol 178(3):687-691

8. Middleton WD, Teefey SA, Reading CC et al (2017) Multi institutional analysis of thyroid nodule risk stratification using the American College of Radiology Thyroid Imaging Reporting and Data System. AJR Am J Roentgenol. 208(6):1331-1341 
9. Ahn SS, Kim EK, Kang DR et al (2010) Biopsy of thyroid nodules: comparison of three sets of quidelines. AJR Am J Roentgenol. 194(1):31-37

10. Kovacevic O, Skurla MS (2007) Sonographic diagnosis of thyroid nodules: correlation with the results of sonographically guided fine-needle aspiration biopsy. J Clin Ultrasound. 35(2):63-67

11. Park JY, Lee HJ, Jang HW et al (2009) A proposal for a thyroid imaging reporting and data system for ultrasound features of thyroid carcinoma. Thyroid 19:1257-1264

12. Na DG, Baek JH, Sung JY et al (2016) Thyroid imaging reporting and data system risk stratification of thyroid nodules: categorization based on solidity and echogenicity. Thyroid 26:562-572

13. Ito Y, Kobayashi K, Tomoda C et al (2005) III-defined edge on ultrasonographic examination can be a marker of aggressive characteristic of papillary thyroid micro carcinoma. World J Surg. 29(8):1007-1012

14. Cappelli C, Pirola I, Cumetti D et al (2005) Is the anteroposterior and transverse diameter ratio of nonpalpable thyroid nodules sonographic criteria for recommending fine needle aspiration cytology? Clin Endocrinal. 63(6):689-693

15. Reading CC, Charboneau JW, Hay ID et al (2005) Sonography of thyroid nodules: a "classic pattern" diagnostic approach. Ultrasound Q. 21(3):157-165

\section{Publisher's Note}

Springer Nature remains neutral with regard to jurisdictional claims in published maps and institutional affiliations.

\section{Submit your manuscript to a SpringerOpen ${ }^{\circ}$ journal and benefit from:}

- Convenient online submission

- Rigorous peer review

- Open access: articles freely available online

High visibility within the field

- Retaining the copyright to your article

Submit your next manuscript at $\boldsymbol{\nabla}$ springeropen.com 